\title{
Linfoma de Burkitt nasofaríngeo
}

\author{
Nasopharyngeal Burkitt lymphoma
}

\author{
Eulalio Alberto Gutiérrez-Rodríguez, ${ }^{*+}$ Manuel Ernesto Arzola-Medrano, ${ }^{\ddagger}$ Laura Elvira García-Labastida ${ }^{\S}$ \\ * Facultad de Medicina Unidad Saltillo, Universidad Autónoma de Coahuila; * Oncología Pediátrica, \\ Hospital del Niño "Dr. Federico Gómez Santos"; ${ }^{\circledR}$ Anatomía Patológica, Servicio de Anatomía Patológica, \\ Hospital Universitario de Saltillo, Universidad Autónoma de Coahuila. Saltillo, Coahuila, México.
}

\begin{abstract}
RESUMEN
Introducción: El linfoma de Burkitt es una neoplasia causada por la proliferación clonal de células B. El objetivo de este trabajo es dar a conocer un caso de linfoma de Burkitt de localización en nasofaringe, lo cual es una presentación muy poco frecuente. Caso clínico: Paciente femenino de ocho años, con padecimiento caracterizado por ronquidos y cambios en la fonación sugestivos de sinusitis crónica. Como parte del estudio, en una tomografía se observó masa de aspecto benigno en nasofaringe. Por nasofibrolaringoscopía se sugiere toma de biopsia. Sin embargo, la paciente evoluciona con disfagia y parálisis facial. El reporte de biopsia fue de linfoma no Hodgkin, de patrón difuso, por lo que se realizan estudios de inmunohistoquímica que determinan linfoma de Burkitt. La paciente fue tratada con quimioterapia con éxito. Conclusión: El linfoma de Burkitt con localización nasofaríngea puede presentar sintomatología inespecífica o simular otras afecciones, por lo cual forma parte del diagnóstico diferencial en problemas de senos paranasales, nariz o de deglución.
\end{abstract}

Palabras clave: Linfoma de Burkitt, linfoma no Hodgkin, pediátrico, nasofaringe.

\section{INTRODUCCIÓN}

El cáncer infantil es una de las primeras causas de muerte en los niños, y en ocasiones representa un desafío establecer el diagnóstico porque la sintomatología puede ser inespecífica. ${ }^{1}$

\footnotetext{
+ Correspondencia: EAGR, lalo_042811@hotmail.com Conflicto de intereses: Los autores declaran que no tienen. Citar como: Gutiérrez-Rodríguez EA, Arzola-Medrano ME, GarcíaLabastida LE. Linfoma de Burkitt nasofaríngeo. Rev Mex Pediatr. 2020; 87(5); 195-200. https://dx.doi.org/10.35366/97174
}

\begin{abstract}
Introduction: Burkitt's lymphoma is a neoplasm caused by the clonal proliferation of B cells. This article aims to present a case of Burkitt's lymphoma located in the nasopharynx, which is a very rare presentation. Clinical case: Eight-year-old female patient with a condition characterized by snoring and changes in speech suggestive of chronic sinusitis. As part of the study, a mass with a benign appearance was observed in the nasopharynx, by tomography. By nasofibrolaryngoscopy, biopsy is suggested. The biopsy report was of non-Hodgkin's lymphoma, with a diffuse pattern, and Burkitt's lymphoma was determined by immunohistochemistry. The patient was successfully treated with chemotherapy. Conclusion: Burkitt lymphoma with nasopharyngeal location can present nonspecific symptoms or simulate other conditions, for which it is part of the differential diagnosis the paranasal sinuses, nose or swallowing diseases.
\end{abstract}

Keywords: Burkitt lymphoma, non-Hodgkin lymphoma, pediatric, nasopharynx.

En general, los linfomas representan el segundo o tercer lugar en frecuencia del total de neoplasias en pacientes menores de 15 años, siendo el linfoma de Burkitt el más común dentro del grupo de linfomas no Hodgkin. En México, el linfoma de Burkitt representa cerca del $11 \%$ del total de linfomas, con una incidencia estimada en 1-2 casos por cada 106 niños con cáncer al año. Estas neoplasias son raras en zona de cabeza y cuello (20-30\%); en esta región, la principal afectación es ganglionar, pero pueden presentarse en nasofaringe, glándulas salivales y senos paranasales. ${ }^{2-5}$ 
El linfoma de Burkitt fue descubierto a principios del siglo XX, siendo Denis Burkitt en 1958 quien proporcionó la descripción detallada de esta patología; además, fue el primer tumor asociado a etiología viral y en demostrarse una translocación cromosómica activadora del oncogén. ${ }^{5-7}$ Se origina por una proliferación clonal de las células tipo B del centro germinal ocasionado principalmente por la translocación $\mathrm{t}(8,14)$, la cual está presente en la mayoría de este tipo de linfomas. ${ }^{7-9}$

Fuera de África existe poca literatura acerca de la presentación de linfomas de Burkitt en la nasofaringe.
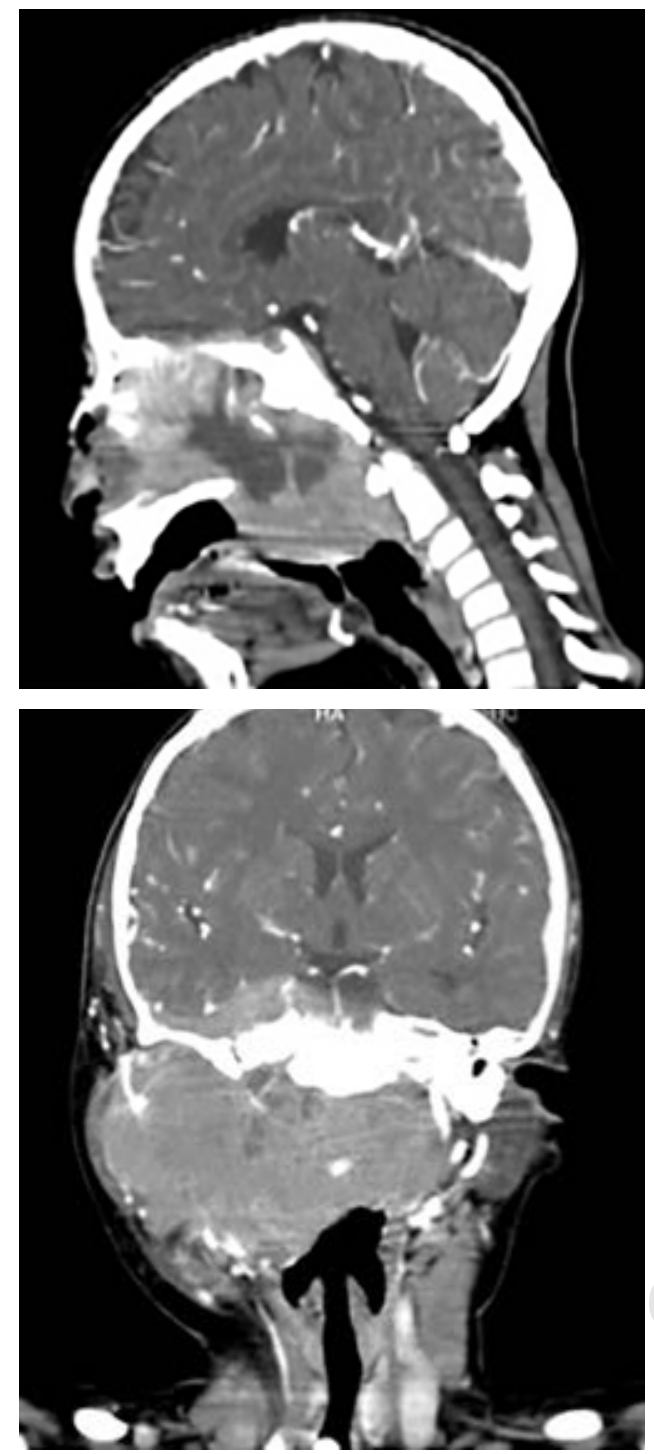

Figura 1: Tomografía axial computarizada que muestra tumoración homogénea isodensa que produce obstrucción a nivel nasofaríngeo.

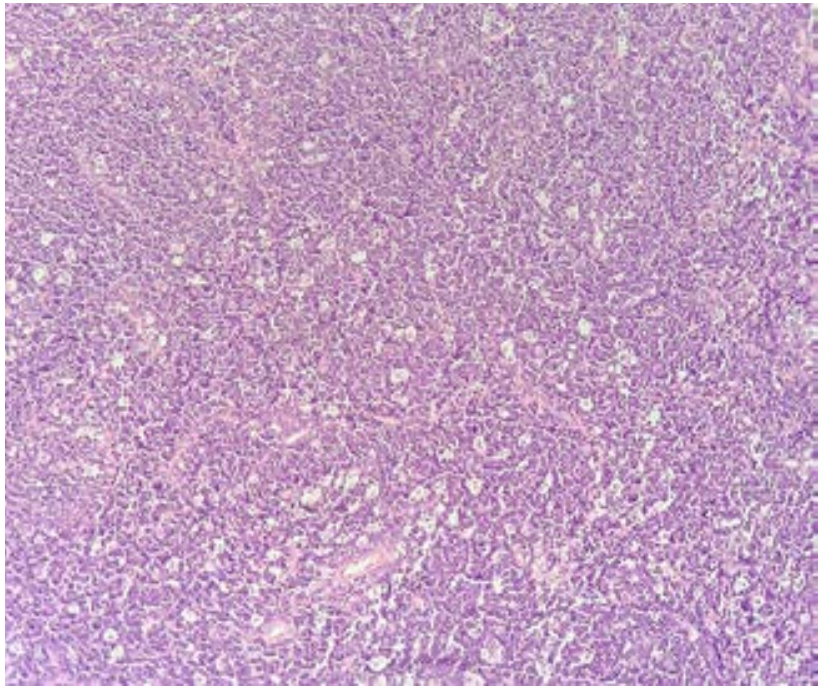

Figura 2: Tinción HyE, vista panorámica 10x, que muestra el clásico patrón de cielo estrellado.

En cuanto a la sintomatología, la mayoría presenta obstrucción nasal, además de rinorrea, cambios en la fonación, sensación de masa, dolor facial y cefalea. Asimismo, en cerca de un tercio puede haber manifestaciones oftalmológicas, siendo la proptosis lo más frecuente. ${ }^{10-13}$

Dado lo raro de la presentación, el objetivo de este trabajo es informar de un paciente pediátrico con linfoma de Burkitt a nivel de la nasofaringe.

\section{PRESENTACIÓN DEL CASO}

Paciente femenino de ocho años de edad, sin antecedentes de importancia. Inició su padecimiento en marzo de 2019, solamente con ronquidos y cambio de la voz. En abril es evaluada por el servicio de Otorrinolaringología, quien solicita una tomografía computarizada (TAC) por probable sinusitis que ha tenido poca respuesta al tratamiento.

La TAC reveló una masa de características benignas, por lo que se solicita TAC sagital y realización de una nasofibrolaringoscopía. En esta última se observó una masa blanquecina de consistencia blanda y friable, que ocupaba la totalidad de la fosa nasal izquierda, con abundante moco hialino a nivel de meato medio, lo cual obstaculizó revisar la nasofaringe. Dado los hallazgos, se programó a toma de biopsia.

En mayo interrumpe actividades escolares y se hospitaliza por cuadros de hematemesis de repetición y disfagia a sólidos. Se realizó angiorresonancia, la cual reportó masa en nasofaringe con extensión a espacios 


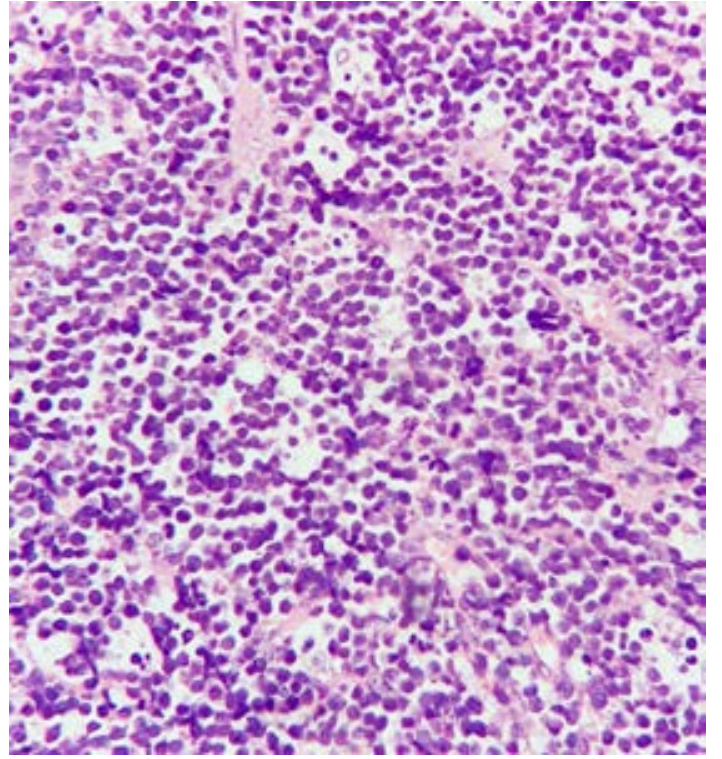

Figura 3: Tinción HyE, acercamiento que muestra los macrófagos con cuerpos apoptóticos.

masticador y parafaríngeo derecho. Se observó también adenopatía cervical ipsilateral, por lo cual se sospechó proceso neoplásico.

En junio la paciente requiere hospitalización por dificultad para la alimentación, por datos de parálisis facial (imposibilidad para levantar la ceja derecha y cerrar los ojos, así como desviación de comisura labial). En una nueva TAC se observó aumento de volumen cervical bilateral, el cual no fue observado previamente, además de un tumor homogéneo isodenso que obstruye totalmente la nasofaringe, infiltrando tejidos blandos de cuello y paladar blando (Figura 1). Se le realiza traqueostomía y gastrostomía para presentar vía aérea que se encontraba parcialmente obstruida.

Se realiza biopsia ganglionar cervical derecho nivel II y biopsia transnasal de tumor primario. El informe histopatológico reporta linfoma no Hodgkin, de patrón difuso, células grandes de alto grado, probable linfoma de Burkitt (Figuras 2 y 3). Se realiza panel de inmunohistoquímica orientado resultando positivo para CD10 (Figura 4A), CD20 (Figura 4B), la Ki67 en 90\% (Figura $5 \mathrm{~A})$ y C-Myc (Figura 6) y presentando negatividad para BCL-2 (Figura 5B) y CD3 (Figura 5C).

En el mismo mes de junio inició tratamiento con quimioterapia bajo el protocolo FAB LMB-96, que incluye vincristina, prednisona, ciclofosfamida, doxorrubicina, metotrexato, citarabina y etopósido. En julio hubo disminución del volumen tumoral a nivel cervical, el cual ya no era palpable (Figura 7).

Durante su tratamiento presentó complicaciones infecciosas asociadas a la administración de quimioterapia como neutropenia febril y colitis neutropénica. Después de nueve meses de tratamiento se consideró que había buena terapéutica, ya que la paciente se encontraba libre de sintomatología y en controles con tomografía por emisión de positrones (PET) no se observaron datos de actividad.

$\mathrm{Al}$ momento del presente reporte la paciente se presenta asintomática. A la exploración física con secuelas de parálisis facial, pero en tratamiento de rehabilitación. Desde el comienzo de la sintomatología, la paciente presentaba un peso de $25.6 \mathrm{~kg}$, el cual disminuyó a $23.2 \mathrm{~kg}$ durante la presentación clínica de la enfermedad. En la actualidad, su peso es de $26.0 \mathrm{~kg}$.
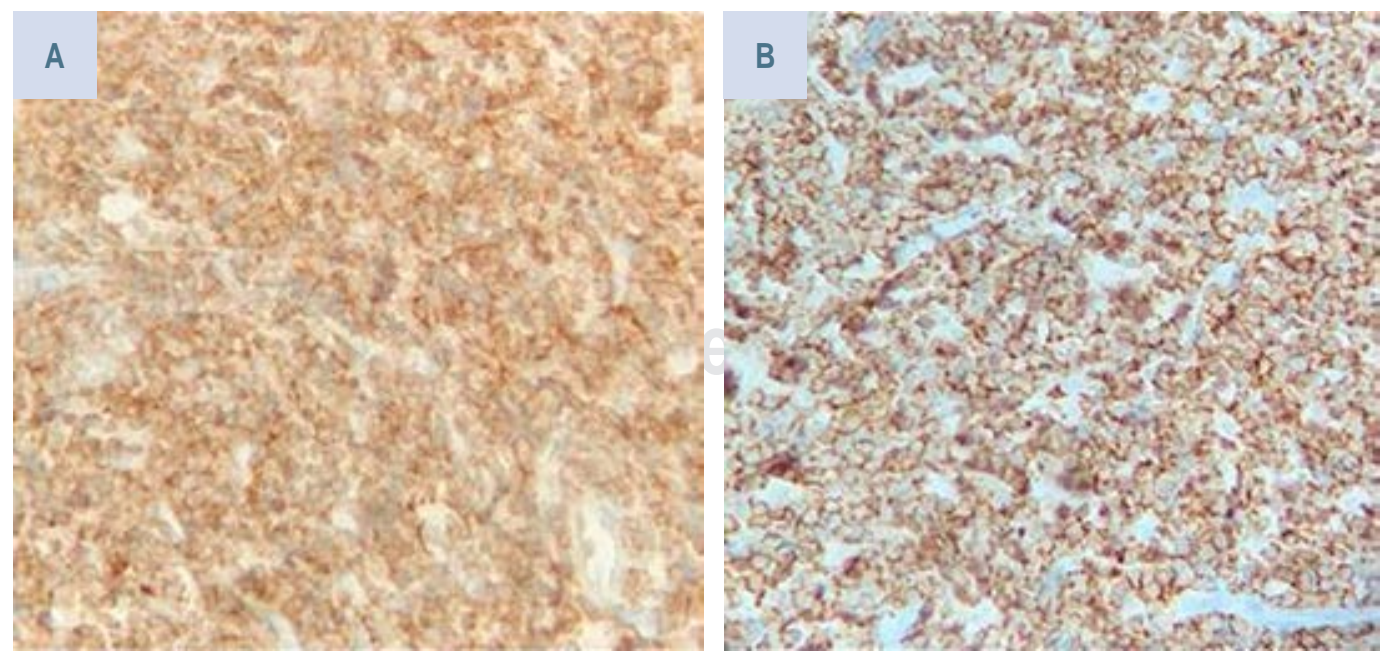

Figura 4:

Tinción de
inmunohistoquímica
con positividad para
CD10 (A) y CD20 (B).




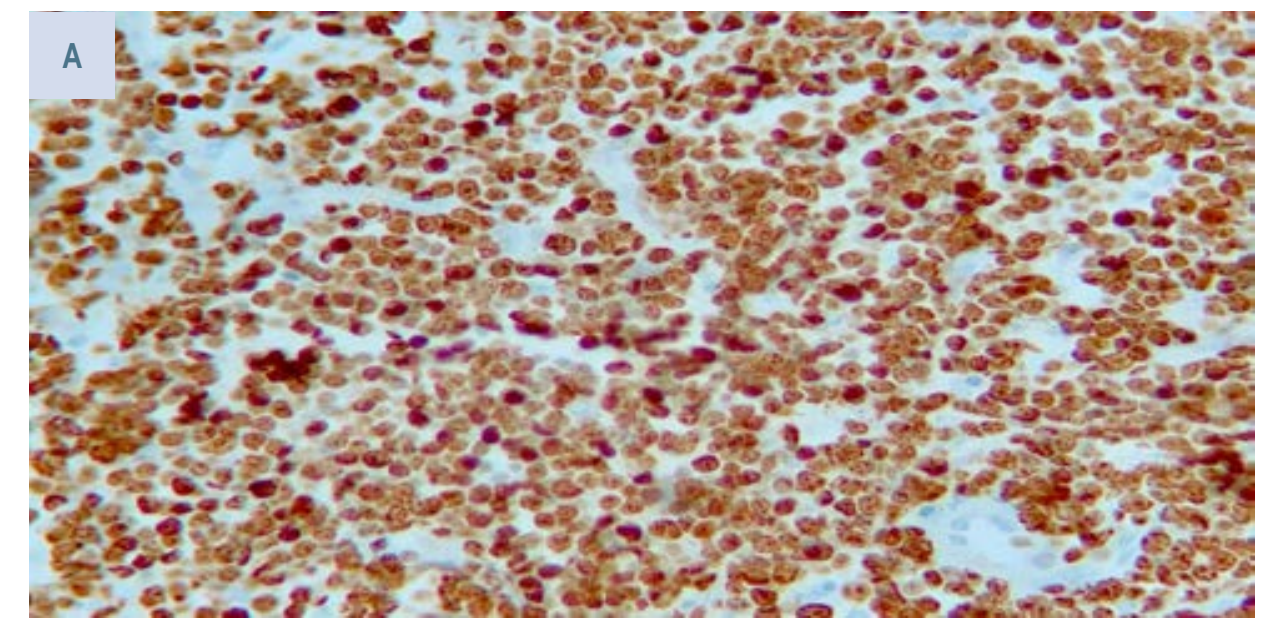

Figura 5:

Tinción de inmunohistoquímica con positividad para Ki67 en $90 \%$ (A) y negatividad para BCL-2 (B) y CD3 (C).

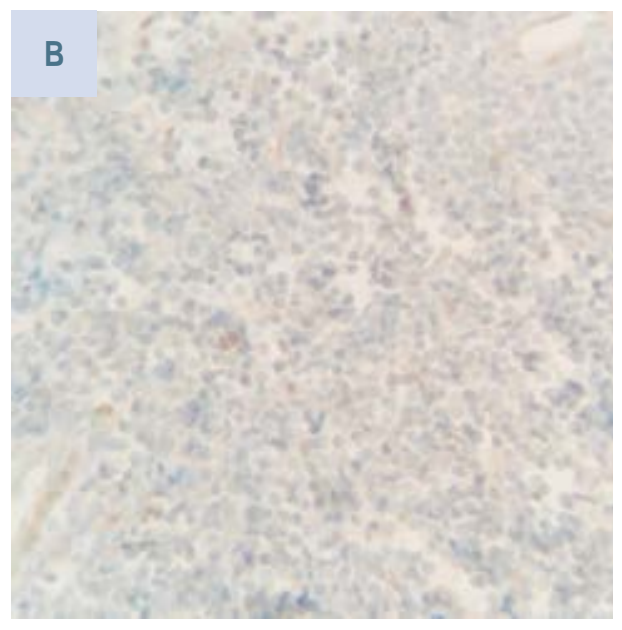

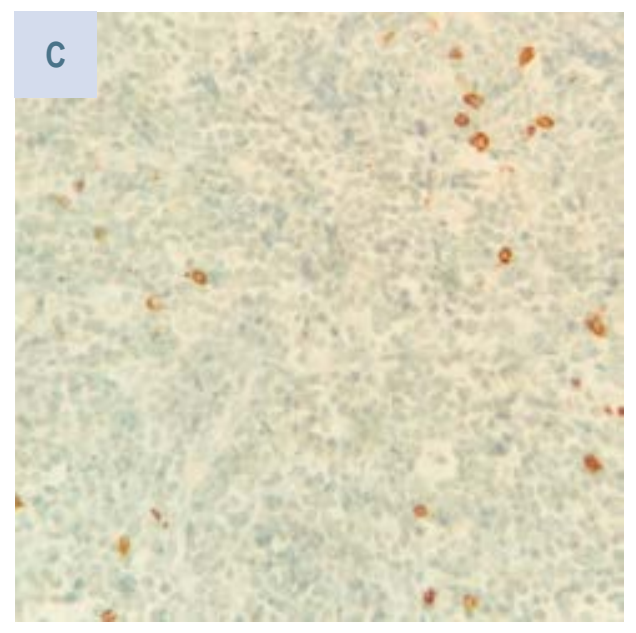

DISCUSIÓN

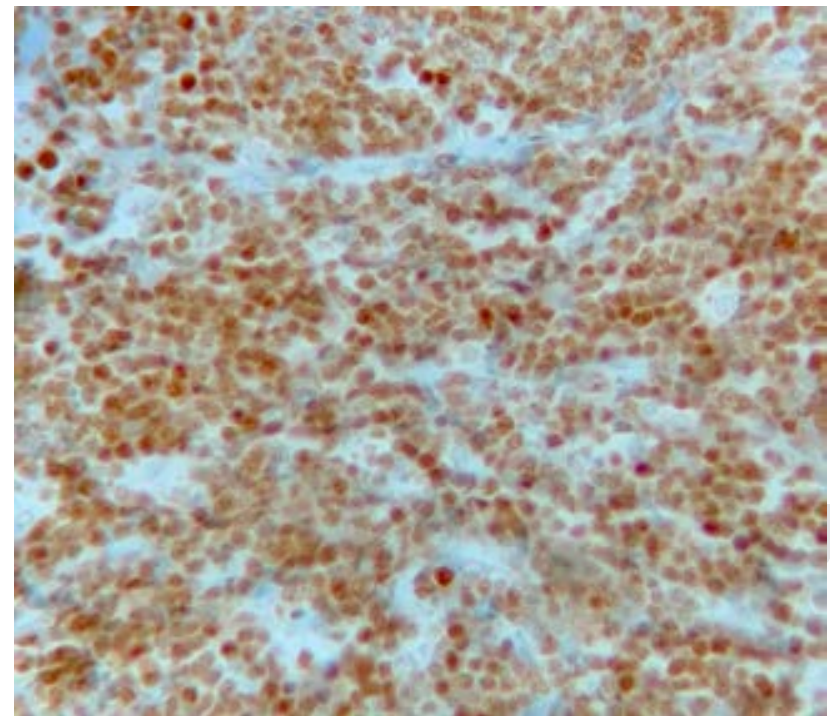

Figura 6: Tinción de inmunohistoquímica con positividad para C-Myc.
El linfoma de Burkitt es el responsable del 13-16\% de los linfomas en edad pediátrica. Existen tres variantes: endémico, esporádico y asociado a inmunodeficiencia, siendo el esporádico, de localización abdominal, el que predomina en México. En el ámbito mundial se reporta un pico de incidencia a los 10 años y con predominio masculino $(2: 1) \cdot{ }^{1-4}$

Dentro de las características clínicas del linfoma de Burkitt variante esporádica, lo más común es su presentación abdominal (50\%) o en médula ósea. Es infrecuente su localización en cabeza y cuello, pero de presentarse en dichas zonas, se presentan como adenopatías cervicales, seguido de localización en maxilar, nasofaringe, mandíbula y amígdalas. ${ }^{6,7}$

Para establecer el diagnóstico se requiere la combinación de parámetros genéticos, histológicos y por inmunofenotipo. Por histología se observan células homogéneas con núcleos ovales con nucléolos basófilos y 

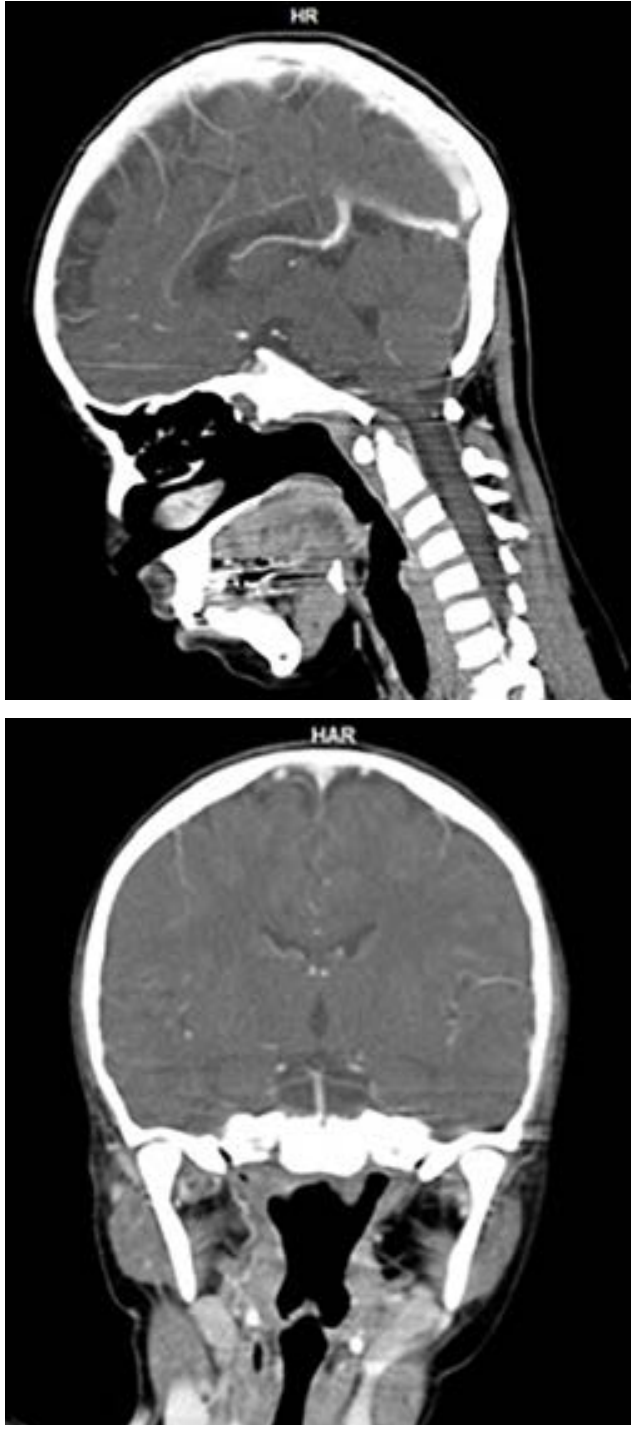

Figura 7: Tomografía axial computarizada que muestra disminución de volumen tumoral a nivel cervical tras el primer ciclo de quimioterapia.

citoplasma abundante y claro. Por la elevada actividad mitótica de estos tumores, los tejidos suelen mostrar el patrón de "cielo estrellado" por la presencia de macrófagos reactivos entre las células linfoides malignas, que contienen restos apoptóticos. Por inmunofenotipo es común que CD10, CD19, CD20, CD22 sean positivos. Además, es necesario que Bcl2 y CD3 sean negativos para descartar otros tipos de linfomas, así como demostrar translocaciones; la translocación $\mathrm{t}(8,14)$ (q2, q32) es la más frecuente. Sin embargo, la dificultad para la realización de pruebas moleculares hace que la histología e inmunohistoquímica sean suficientes para el diagnóstico. ${ }^{7-9}$

En los auxiliares laboratoriales destacan la elevación de la deshidrogenasa láctica (50\%) y del ácido úrico $(25 \%)$ pero son inespecíficos. Las técnicas de imagen son de utilidad para sospecha diagnóstica, localización exacta, realización de biopsia y control terapéutico. $^{7-9}$

Existe poca bibliografía con casos similares; sin embargo, en las publicaciones previas se resalta la importancia de la sintomatología asociada a la localización anatómica, como la afección rinosinusal y los cambios en fonación y ronquidos. También hay síntomas neurológicos que coinciden, principalmente la parálisis facial. A diferencia de otros casos, nuestra paciente no mostró sintomatología oftalmológica, lo cual se ha reportado hasta en el $33 \%$ de los pacientes. ${ }^{10-12}$

En cuanto al tratamiento, la ciclofosfamida es el pilar de la quimioterapia, pero asociado a otros fármacos para favorecer la remisión. La duración de la remisión es muy variable, llegando a ser en promedio de 18 meses. En general, para el linfoma de Burkitt se puede esperar supervivencia de cerca del $90 \%$ a los tres años, cuando se detecta en etapas tempranas. El pronóstico se puede ver empobrecido por el retraso en el diagnóstico; en estadio IV, los pacientes con linfomas no Hodgkin la supervivencia puede ser hasta del 48\%. Sin embargo, los niños en comparación con la población adulta presentan mejores tasas de supervivencia. ${ }^{13,14}$

\section{REFERENCIAS}

1. Johnston W, Erdmann F, Newton R, Steliarova-Foucher E, Schüz J, Roman E. Childhood cancer: Estimating regional and global incidence. Cancer Epidemiol. 2020; 101662. doi: 10.1016/j. canep.2019.101662.

2. Rendón-Macías ME, Riojas-Garza A, Pachuca-Vazquez A, Martínez-Ezquerro JD, Fajardo-Gutiérrez A. Non-Hodgkin lymphoma epidemiology in children from 2 socioeconomic regions in Mexico. J Pediatr Hematol Oncol. 2020; 42: 292-298. doi: 10.1097/mph.0000000000001635.

3. Fajardo-Gutiérrez A, Juárez-Ocaña S, González-Miranda G, Palma-Padilla V, Carreón-Cruz R, Ortega-Alvárez MC et al. Incidence of cancer in children residing in ten jurisdictions of the Mexican Republic: importance of the Cancer registry (a populationbased study). BMC Cancer. 2007; 7: 68. doi: 10.1186/1471-24077-68.

4. Rendón-Macías ME, Valencia-Ramón EA, Fajardo-Gutiérrez A. Clinical and epidemiological characteristics of Burkitt lymphomas in pediatric patients from two defined socioeconomic regions in Mexico. J Trop Pediatrs. 2017; 63(4): 253-259. doi: 10.1093/tropej/ fmw082.

5. Rivera-Luna R, Martínez-Guerra G, Borrego-Román R, RiveraMárquez $\mathrm{H}$. Burkitt's lymphoma: experience at the Instituto 
Nacional de Pediatria, Mexico City. Am J Pediatr Hematol Oncol. 1986; 8: 183-190. doi: 10.1097/00043426-198623000-00003.

6. Magrath I. Epidemiology: clues to the pathogenesis of Burkitt lymphoma. Br J Haematol. 2012; 156: 744-756. doi: 10.1111/j.1365-2141.2011.09013.x.

7. Magrath I. B-Cell Lymphoma/Burkitt lymphoma. Pediatric Lymphomas Pediatric Oncology. 141-174. doi: 10.1007/978-3540-68753-5_7.

8. Mundo L, Ambrosio MR, Raimondi F, Porro LD, Guazzo R, Mancini $V$ et al. Molecular switch from MYC to MYCN expression in MYC protein negative Burkitt lymphoma cases. Blood Cancer Journal. 2019; 9. doi: 10.1038/s41408-019-0252-2.

9. Agarwal R, Lade S, Liew D, Rogers TM, Byrne D, Feleppa $F$ et al. Role of immunohistochemistry in the era of genetic testing inMYC-positive aggressive B-cell lymphomas: a study of 209 cases. J Clin Pathol. 2015; 69: 266-270. doi: 10.1136/ jclinpath-2015-203002.

10. Hong X, Khalife S, Bouhabel S, Bernard C, Daniel SJ, Manoukian $\mathrm{JJ}$ et al. Rhinologic manifestations of Burkitt lymphoma in a pediatric population: Case series and systematic review. Int $\mathrm{J}$ Pediatr Otorhinolaryngol. 2019; 121:127-136. doi: 10.1016/j.jporl.2019.03.013.

11. Xuereb G, Borg J, Apap K, Borg C. The snoring 2-year-old boy: a case of primary nasopharyngeal Burkitt's lymphoma. BMJ Case Reports. 2020; 13: e233536. doi: 10.1136/bcr-2019233536.

12. Pedraza RM, Arboleda LP, Sánchez-Romero C, Quiñones JA, Tovar CD, Henao JR et al. Intraoral EBV-positive sporadic Burkitt lymphoma in an elderly patient with bilateral presentation. Autopsy Case Rep. 2019; 9(4): e2019117. doi: 10.4322/acr.2019.117.

13. Mukhtar F, Boffetta P, Risch HA, Park JY, Bubu OM, Womack L et al. Survival predictors of Burkitts lymphoma in children, adults and elderly in the United States during 2000-2013. Int J Cancer. 2017; 140: 1494-1502. doi: 10.1002/ijc.30576.

14. Kalisz K, Alessandrino F, Beck R, Smith D, Kikano E, Ramaiya NH et al. An update on Burkitt lymphoma: a review of pathogenesis and multimodality imaging assessment of disease presentation, treatment response, and recurrence. Insights Imaging. 2019: 56. doi: 10.1186/s13244-019-0733-7. 